team. Majority (75\%) of the handovers were presented by registrars/junior trainees with only 35\% receiving any feedback.

SBAR (Situation, Background, Assessment and Recommendation) method was only used for $42 \%$ of handovers. Majority $(70 \%)$ of the handovers were conducted with the aid of printed sheets, which included: patient demographics (83\%), presenting complaints $(85 \%)$, investigations, results and treatment plans (83\%). Only $11 \%$ of handovers were done electronically. Handovers had allocated start times (96\%) with designated places (89\%) close to area of work. However only $63 \%$ of the handovers started on time, $20 \%$ were free from distractions by allied professionals and just $5 \%$ were 'bleep' free. $68 \%$ had some educational activity within the time allocated in the handover. WPBAs were initiated or completed in only $11 \%$ of handovers. Overall $91 \%$ of trainees felt that the quality of handover was either average or good.

Conclusions The findings from our survey suggest that the quality of handovers is variable. Handovers should have a structured approach and free from distractions to ensure safety and continuity of care. Incorporating formal teaching and WPBA's could help develop the role of handovers.

\section{G23(P) EDUCATION AND TRAINING USING AN INNOVATIVELY ADAPTED MANIKIN: SIMPLE, AFFORDABLE, FEASIBLE AND EFFECTIVE (SAFE)}

doi:10.1136/archdischild-2013-304107.036

${ }^{1} \mathrm{NB}$ Soni, ${ }^{1} \mathrm{~A}$ Cox, ${ }^{2} \mathrm{E}$ McLeod, ${ }^{3} \mathrm{~A}$ Patel, ' $\mathrm{C}$ Harrison. ${ }^{1} \mathrm{NICU}$, Lancashire Women and Newborn Centre, Burnley, UK; ${ }^{2}$ North-West Deanery, UK; ${ }^{3} E B M E$ Department, East Lancashire Hospitals NHS Trust, UK

Introduction Hi-fidelity manikins are often used in simulation courses. However they are very expensive and some of the skills like drainage of pneumothorax or insertion of chest-drains/rectal probes cannot be demonstrated on these manikins as they are fully loaded with various electronic equipment inside them and puncturing will damage these expensive manikins. Hence our team developed a multi-purpose, low cost, Low-fidelity manikin where wide variety of neonatal practical skills can be practised.

Aims and methods Aim was not only to create simulation of real clinical situations but also to teach practical skills and build the concept of team working. ALS Manikin was modified as below:

1. An innovatively-designed container with red fluid was placed in abdominal cavity and connected to synthetic umbilical cord. Umbilical arterial line was connected through an innovatively-designed simulator transducer box producing arterial wave form with feasibility to vary BP using solenoid valve.

2. Manikin's chest was drilled between ribs and lungs were made from Nitrile gloves. These lungs on connecting to flow metre were able to show positive trans-illumination test and provided air filled lungs for needle thoracocentesis and chestdrain insertion.

3. Manikin's bottom was drilled for rectal probe insertion. Thermistor from rectal probe was removed and connexions made to an innovative resistance box. With the help of Ohms Law principle, we were able to replicate any rectal temperature with an accuracy of $0.1^{\circ} \mathrm{C}$.

Following above adaptations, regular simulation sessions were initiated for:

1. Trainees to undertake practical skills like emergency needle thoracocentesis, pigtail chest drain insertion, umbilical lines insertion/sampling.

2. Train nursing staff with rectal probe insertion, familiarise with connexions of chest-drain and umbilical lines.

3. Both medical and nursing staff to work in team to develop effective communication.

\section{Results}

1. All rotating registrars have had exposure to pigtail chestdrain insertion in simulation setting and subsequently went on to undertake these skills in NICU on real patients with greater confidence.

2. Improved team working observed between doctors and nursing staff on NICU

Conclusions Our method of manikin manipulation is innovative, affordable and effective and can be implemented in any hospital setting to teach practical neonatal skills, improve team working, enhance competency at performing practical skills and work with increased confidence.

\section{Clinical Genetics Group/British Society of Paediatric Dermatology}

\section{G24 CAPILLARY MALFORMATIONS - ARTERIOVENOUS MALFORMATIONS/ARTERIOVENOUS FISTULA SYNDROME (CM-AVM SYNDROME): AN UNDER RECOGNISED CLINICAL ENTITY?}

doi:10.1136/archdischild-2013-304107.037

II Thanopoulou, ${ }^{2} \mathrm{~S}$ Bhate, ${ }^{3} \mathrm{~N}$ Burrows, ${ }^{4} \mathrm{~J}$ Berg, ${ }^{1} \mathrm{M}$ Glover. ${ }^{1}$ Paediatric Dermatology, Great Ormond Street Hospital, London, UK; ${ }^{2}$ Paediatric Neurology, Great Ormond Street Hospital, London, UK; ${ }^{3}$ Dermatology, Addenbrookes Hospital, Cambridge, UK; ${ }^{4}$ Clinical Genetics, Ninewells Hospital And Medical School, Dundee, UK

Background Hereditary Hemorrhagic telangiectasia (HHT) tends to be the first condition to be considered in the differential diagnosis of patients presenting with high flow vascular malformations in combination with cutaneous vascular lesions. However, particularly in the paediatric population, capillary malformation-arteriovenous malformation syndrome (CM-AVM) due to RASA-1 mutation ${ }^{1}$ is more likely.

Aims To present the clinical features of three patients with CMAVM syndrome, promote knowledge of this condition and aid prompt diagnosis.

Methods Clinical examination, detailed family history, imaging (ultrasound, MRI, angiography) and genetic testing.

Results Patient 1 was born with a large vascular mass affecting the right side of the face and multiple cutaneous capillary malformations. Patient 2 had a spinal AV fistula and two vascular stains. Patient 3 presented with an intracranial haemorrhage secondary to a parietal AVM and was noted to have several cutaneous vascular lesions. Patients 2 and 3 were referred to the dermatology team as suspected HHT. The cutaneous vascular lesions present in all three patients were consistent with capillary malformations (in keeping with a diagnosis of (M-AVM) and were not typical of telangiectases.

Conclusion In patients with high flow CNS vascular lesions, it is crucial to establish the precise nature of cutaneous vascular lesions in order to request appropriate genetic testing and screening of relatives.

\section{REFERENCE}

1. Laurence M Boon, Nicole Revencu, Miikka Vikkula, Université catholique de Louvain, Brussels, Belgium

RASA1 MUTATIONS AND VEIN OF GALEN ARTERIAL MALFORMATIONS

doi:10.1136/archdischild-2013-304107.038

${ }^{1} \mathrm{AM}$ Heuchan, ${ }^{2} \mathrm{~S}$ Joss, ${ }^{3} \mathrm{~J}$ Berg, ${ }^{4} \mathrm{M}$ Suri, ${ }^{5} \mathrm{~J}$ Bhattacharya. ${ }^{1}$ Neonatal Medicine, Royal Hospital for Sick Children, Glasgow, UK; ${ }^{2}$ Clinical Genetics, Royal Hospital for Sick Children and Southern General Hospital, Glasgow, UK; ${ }^{3}$ Clinical Genetics, Ninewells Hospital, Dundee, UK; ${ }^{4}$ Clinical Genetics, Nottingham University Hospital, Nottingham, UK; ${ }^{5}$ Neuroradiology, Southern General Hospital, Glasgow, UK 
Aims Vein of Galen arterial malformation (VGAM) is a rare high flow cerebral arteriovenous malformation which most commonly presents with cardiac failure in infancy. VGAM is considered to be a sporadic disorder, with a population incidence of 1 in 100,000. No genetic basis or increased risk of recurrence within affected families has been identified previously. Recently, RASA1 gene mutations have been identified as causative in the autosomal dominant capillary malformation arteriovenous (AV) malformation (CM-AVM) syndrome, a condition presenting with multiple skin AV malformations. A large European study of affected kindreds identified associated non-cutaneous AV malformations and, amongst 140 individuals, identified two cases of VGAM, raising the possibility of a genetic basis for this condition ${ }^{1}$. The aim of the present study was to assess the frequency and type of RASA1 mutations in a population presenting with VGAM malformation.

Methods A National Centre for VGAM treatment obtained consent for RASA 1 mutation analysis for all cases presented to the service from January 2011. Genomic DNA was obtained from blood samples and the 25 exons of the RASA1 gene were sequenced for each patient.

Results RASA1 analysis has been undertaken for 11 cases and four were positive for mutations: c.2912 T > C(missense), c.2125C > T (truncated) and C.2119C $>\mathrm{T}$ (missense) (two cases). The two cases with the C.2119C > T mutation were siblings. One case, with the c.2125C > T mutation, developed the typical CM-AVM rash.

Conclusions RASA1 mutations are strongly associated with VGAM and are biologically plausible causative mutations. The autosomal dominant inheritance of this mutation, has a significant implication for counselling affected families.

\section{REFERENCE}

1. Revencu N, Boon L, Mulliken J et al ParkesWeber Syndrome, Vein of Galen Aneurysmal Malformation, and Other Fast-Flow Vascular Anomalies Are Caused by RASA1 Mutations. Human Mutation 29(7),959-965,2008

\section{G26 LYMPHATIC DISORDERS IN NOONAN SYNDROME}

doi:10.1136/archdischild-2013-304107.039

SJ Joyce, S Mansour. Medical Genetics, St George's, University of London, London, UK

Aims To investigate the lymphatic phenotype in Noonan syndrome with reference to the medical literature

Methods Notes from patients with Noonan syndrome attending a lymphoedema clinic were located via the Geneworks database by searching for "Noonan syndrome" and then examining paper notes of those patients who had lymphoedema listed as a feature. The patients' letters and results stored in the Electronic Patient Register were also accessed and information on lymphatic abnormalities and molecular results was gathered and analysed. Images and results of lymphoscintigraphy were obtained from iSite Enterprise.

Results $7 / 582$ Noonan syndrome patients (1.2\%) had lymphoedema listed as a characteristic, and were included in this study.

The current age of patients ranged from 6 to 37 years. Onset of lymphoedema ranged from birth to 27 years, with a mean of 10.3 years.

5/7 patients had swelling of the lower limbs and genitalia. 5/7 had systemic involvement (intestinal lymphangiectasia, chylous reflux or chylothorax). 4/7 had genital lymphorrhoea.

Conclusion This study suggests that severe lymphoedema is a less common feature of Noonan syndrome than previous reports have indicated. Mild lymphoedema in the remaining patients, however, cannot be excluded. Within the patient group studied, a consistent pattern of lymphatic abnormality was seen: lower limb lymphoedema with variable age of onset, and genital involvement with chylous reflux.

\section{G27 THE LYMPHATIC PHENOTYPE IN TURNER SYNDROME: AN EVALUATION OF PATIENTS PRESENTING TO THREE SPECIALIST PRIMARY LYMPHOEDEMA CLINICS AND LITERATURE REVIEW}

doi:10.1136/archdischild-2013-304107.040

${ }^{1} \mathrm{G}$ Atton, ${ }^{3} \mathrm{~K}$ Gordon, ${ }^{1} \mathrm{G}$ Brice, ${ }^{2} \mathrm{~V}$ Keeley, ${ }^{2} \mathrm{~K}$ Riches, ${ }^{4} \mathrm{P}$ Mortimer, ${ }^{1} \mathrm{~S}$ Mansour. 'SW Thames Regional Genetics Unit, St George's, University of London, London, UK; ${ }^{2}$ Nightingale Macmillan Unit, Derby Hospitals NHS Foundation Trust, Derby, UK; ${ }^{3}$ Department of Dermatology, St. George's Hospital NHS Trust, London, UK; ${ }^{4}$ Cardiac and Vascular Sciences (Dermatology), St George's University of London, London, UK

Aims This study aimed to analyse the medical records of a cohort of 19 Turner Syndrome patients attending three specialist primary lymphoedema clinics to elucidate the key features of the lymphatic phenotype of Turner Syndrome and provide vital insights into its diagnosis, progression and management. Lymphoedema of the hands, feet and cervical region is a common and key diagnostic indicator of Turner Syndrome, present in $>60 \%$ of patients, though is poorly described in the literature.

Methods The study sample of 19 female patients was obtained from specialist primary lymphoedema clinics at three major centres and located by identifying all patients with Turner Syndrome and lymphoedema from hospital databases. Patient and genetic notes were identified through the use of patient-specific numerical identifiers. These notes were thoroughly analysed and examined and any important information inserted into a spreadsheet pro forma.

Results The majority of patients presented at birth with 4-limb lymphoedema which often resolved in early childhood but frequently recurred in later childhood. There was 1 case of systemic involvement (e.g. intestinal or pulmonary lymphangiectasia). The swelling was confined to the legs and hands with no facial or genital swelling. The most significant discovery from this research was a pattern observed from the lymphoscintigraphy results, which suggested that the lymphatic phenotype of Turner Syndrome may be due to lymphatic functional hypoplasia of lymphatic tracts.

Conclusion Turner Syndrome frequently presents at birth with 4-limb lymphoedema which often resolves in early childhood but may recur at any age. The lymphoscintigraphy results suggested that the lymphatic phenotype in Turner Syndrome may be due to lymphatic functional hypoplasia, a new perspective which may highlight the importance of this test as a baseline assessment of lymphoedema in Turner Syndrome patients.

\section{G28 A NOVEL MISSENSE MUTATION IN KERATIN 1 UNDERLYING CLINICALLY MILD EPIDERMOLYTIC ICHTHYOSIS MIMICKING EPIDERMOLYSIS BULLOSA SIMPLEX SUPERFICIALIS}

doi:10.1136/archdischild-2013-304107.041

${ }^{1} \mathrm{Al}$ MacKenzie, ${ }^{2} \mathrm{PJ}$ Dopping-Hepenstal, ${ }^{2} \mathrm{~L}$ Ozoemena, ${ }^{2} \mathrm{~L}$ Liu, ${ }^{3} \mathrm{~K}$ Stone, ${ }^{3} \mathrm{MA}$ Simpson, ${ }^{4} \mathrm{JA}$ McGrath, ${ }^{1} \mathrm{AE}$ Martinez, ${ }^{1,5} \mathrm{JE}$ Mellerio. 'Department of Dermatology, Great Ormond Street Hospital for Children NHS Foundation Trust, London, UK; ${ }^{2}$ National Diagnostic Epidermolysis Bullosa Laboratory, St John's Institute of Dermatology, GSTS Pathology, St Thomas' Hospital, London, UK: ${ }^{3}$ Department of Medical and Molecular Genetics, King's College, London, UK; ${ }^{4}$ Genetic Skin Disease Group, St John's Institute of Dermatology, King's College, London, UK; ${ }^{5}$ St John's Institute of Dermatology, Guy's and St Thomas NHS Foundation Trust, London, UK

Inherited skin peeling may be autosomal recessive (AR) or autosomal dominant (AD). When $A R$, this can be localised, as in acral peeling skin syndrome (APSS); or generalised, as in peeling skin syndrome (PSS) types A (non-inflammatory) and B (inflammatory). When $\mathrm{AD}$, this can present in association with ichthyosis as either epidermolytic ichthyosis (EI) or superficial epidermolytic ichthyosis 